\title{
Large Hadron Collider starts doing science again
}

Particle collisions at record energies will push the boundary of human knowledge.

\section{Elizabeth Gibney}

03 June 2015

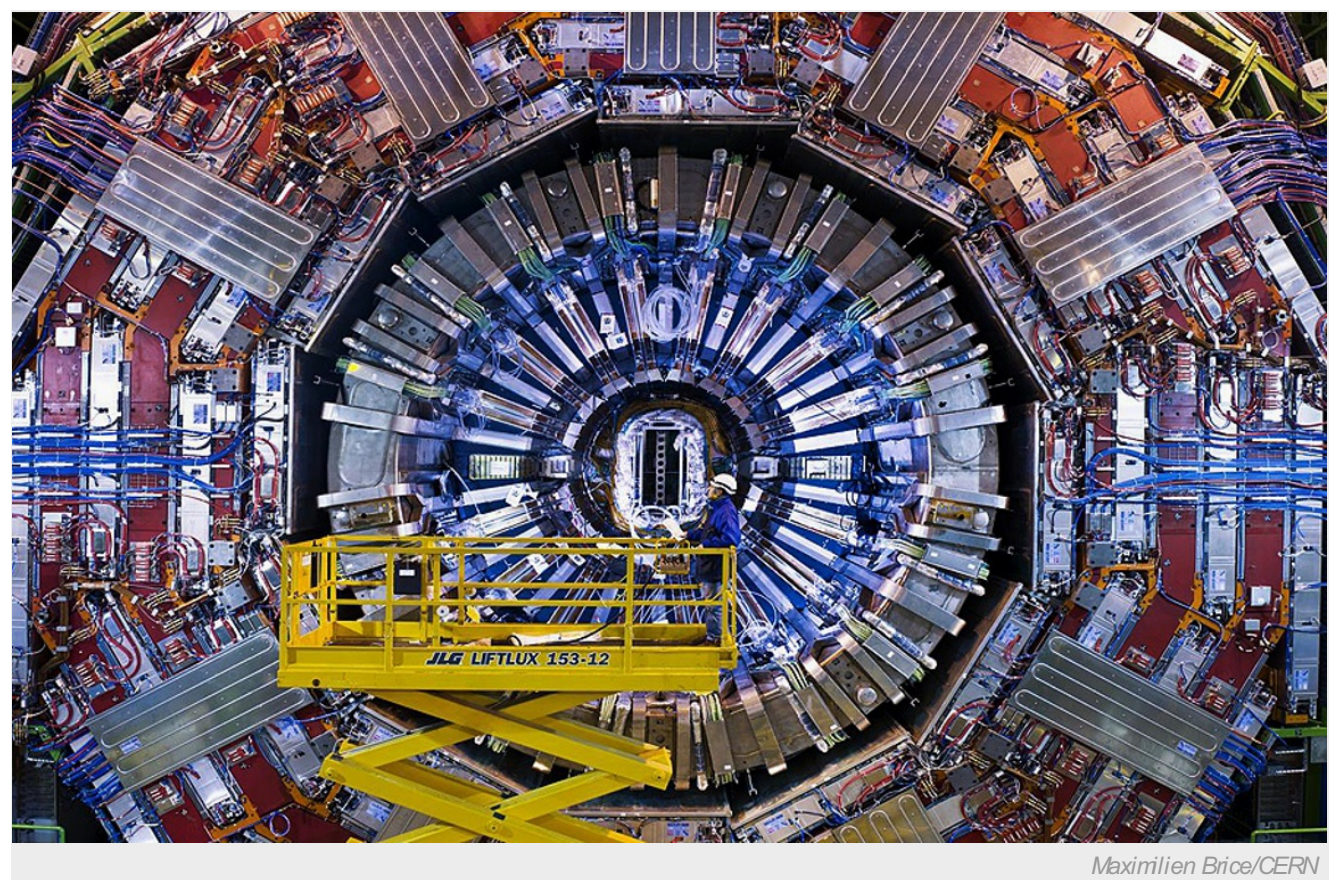

The LHC is smashing protons together at a higher energy than ever before.

The highest-energy collisions ever seen at the Large Hadron Collider (LHC) are now producing data for science.

Teams at CERN, Europe's particle-physics laboratory near Geneva, Switzerland, have spent two years upgrading what was already the world's most powerful particle accelerator. At 10.40 local time this morning (3 June), they officially set the newly supercharged collider running.

Physicists can now smash together bunches of protons at a record energy of 13 teraelectronvolts (TeV) and will soon collide a billion pairs of protons per second - almost double the previous rate. The machine was switched off on 14 February 2013 after an initial period — dubbed run 1 - marked by the discovery of the Higgs boson.

The first beams of protons following the shutdown circled around the 27-kilometre ring in early April, but at low energies. Since then, physicists have worked to check mechanisms designed to protect the machine and to calibrate the beams, before increasing the LHC's energy and bringing its four main experiments fully online.

On 3 June, the collisions started in earnest, with all four detectors collecting data for analysis. "At this stage, the actual number of colliding bunches is rather small, but the number will be progressively increased in the coming months," says Paul Collier, head of beams at CERN.

With more bunches the data will flow in at a faster rate, he adds. His team hopes to increase the collision energy to 14 TeV eventually, before switching off again in 2018 for more upgrades. "At this stage, everything looks good for run 2 of the LHC and, hopefully, a bumper harvest of data at the new operational energy."

\section{Dance of the protons}

When protons collide, part of their energy is converted into mass, creating showers of new particles. Physicists hope that the extra firepower of the upgraded LHC will unearth phenomena that do not fit with the standard model of particle physics, the best description of fundamental particles and their interactions that scientists have right now. This could allow them to answer long-standing questions 
that this model cannot explain, such as what dark matter is, and why the Higgs boson, discovered at the lab in 2012 , is inexplicably light.

A popular theory known as supersymmetry could provide an explanation for these questions, but data from the LHC's first run ruled out many of the simplest versions of the theory. More data and higher-energy collisions could provide evidence for more-complex versions.

"With the additional energy and collision rate of the upgraded LHC, we expect to investigate previously unexplored regions of human knowledge," says Don Lincoln, a physicist at the Fermi National Accelerator Laboratory near Batavia, Illinois, and a member of the CMS experiment at CERN. "We'll search for new ultimate building blocks of the Universe much smaller than before, and cut in half the time after the Big Bang of which we know nothing."

In some cases, it will take only a few days' worth of data to advance on conclusions drawn from the first run. But some questions will take years to answer, adds Lincoln. "I am very confident that we'll be publishing interesting papers almost immediately but, unless we get very lucky, it will be quite a while until we can reasonably hope to make a big discovery," he says.

Nature I doi:10.1038/nature.2015.17659 